\title{
Bi-directional WDM transmission by use of SOAs as inline amplifiers without isolators
}

Jeppesen, Palle; Yu, Jianjun

Published in:

Proceedings of Optical Fiber Communication Conference and Exhibit, 2001

Link to article, DOI:

10.1109/OFC.2001.928508

Publication date:

2001

Document Version

Publisher's PDF, also known as Version of record

Link back to DTU Orbit

Citation (APA):

Jeppesen, P., \& Yu, J. (2001). Bi-directional WDM transmission by use of SOAs as inline amplifiers without isolators. In Proceedings of Optical Fiber Communication Conference and Exhibit, 2001 (Vol. 3)

https://doi.org/10.1109/OFC.2001.928508

\section{General rights}

Copyright and moral rights for the publications made accessible in the public portal are retained by the authors and/or other copyright owners and it is a condition of accessing publications that users recognise and abide by the legal requirements associated with these rights.

- Users may download and print one copy of any publication from the public portal for the purpose of private study or research.

- You may not further distribute the material or use it for any profit-making activity or commercial gain

- You may freely distribute the URL identifying the publication in the public portal 
WDD58-1

\title{
Bi-directional WDM Transmission by Use of SOAs as Inline Amplifiers Without Isolators \\ Jianjun Yu and Palle Jeppesen
}

Research Center COM, Technical University of Denmark, Building 349, Lyngby DK-2800, Denmark

Telephone: 454525 3782, Fax: 454593 6581, email: jy@com.dtu.dk

\begin{abstract}
Error-free bi-directional transmission of $8 \times 10 \mathrm{~Gb} / \mathrm{s}$ signals over two inline SOAs is realized for the first time. It is demonstrated that SOAs can be used for inline amplifiers in bidirectional multi-wavelengths transmission systems at $10 \mathrm{~Gb} / \mathrm{s}$ without any isolator.
\end{abstract}




\title{
Bi-directional WDM Transmission by Use of SOAs as Inline Amplifiers Without Isolators
}

\author{
Jianjun Yu and Palle Jeppesen \\ Research Center COM, Technical University of Denmark, Building 349, Lyngby DK-2800, Denmark \\ Telephone: 45 4525 3782, Fax: 454593 6581, email: jy@com.dtu.dk
}

\section{Introduction}

The SOA is a promising candidate for cascaded optical fiber systems because of the coverage of the entire fiber transmission window and the possibilities for integration and low cost [1-7]. Bi-directional transmission can utilize efficiently the optical fiber transmission bandwidth and reduce the complexity and cost of optical transmission systems and networks. SOAs as optical switches or in-line amplifiers are very suitable for bidirectional transmission systems and networks because SOAs do not need any optical isolators as often used in case of EDFAs. We have demonstrated earlier that an SOA can be used for bi-directional $16 \times 10 \mathrm{~Gb} / \mathrm{s} \mathrm{WDM}$ gating operation at $10 \mathrm{~Gb} / \mathrm{s}$ by use of holding light injection. In this paper, we will demonstrate that it is possible to transmit $8 \times 10 \mathrm{~Gb} / \mathrm{s}$ WDM signals over two in-line SOAs without any isolator. The low input power dynamic range and high XGM induced in multi-channel gating due to gain saturation and XGM respectively [3], have been overcome with the introduction of gain control schemes such as laser gain clamping [4], polarization multiplexing technique (PMT) [5] and holding light injection [6,7]. Theoretical investigation [4] has shown that a four-channel system at $10 \mathrm{~Gb} / \mathrm{s}$ can not be cascaded over 3 SOAs by using small signal injection nor over 6 GC-SOAs by using laser gain clamping with passive and active DBR regions at $1 \mathrm{~dB}$ penalty, mainly due to extinction ratio (ER) degradation for the SOA gates and limited relaxation frequency for the GC-SOA gates. In order to overcome the limited relaxation frequency of GC-SOAs with laser gain control, the methods of holding light injection and PMT can be used. However, PMT involves a complex transmitter. Holding light injection has a simple configuration.

\section{Experimental setup and results}

Fig. 1 shows the measured small signal gain characteristics of two SOAs for an input signal power of -15 $\mathrm{dBm}$. The gain peak wavelengths of SOA1 and SOA2 are 1550 and $1544 \mathrm{~nm}$, respectively. The SOA1 gain is reduced $2.2 \mathrm{~dB}$ when the wavelength is changed from 1550 to $1560 \mathrm{~nm}$, while the SOA2 gain is reduced $6 \mathrm{~dB}$ within the same wavelength range; this shows that SOA2 has a rather un-flattened small signal gain. The measured saturation output powers of SOA1 and SOA2 are 8.9 and $6.7 \mathrm{dBm}$, respectively.

\section{A. $4 \times 10 \mathrm{~Gb} / \mathrm{s}$ bi-directional $44 \mathrm{~km}$ SMF transmission with two in-line SOAs}

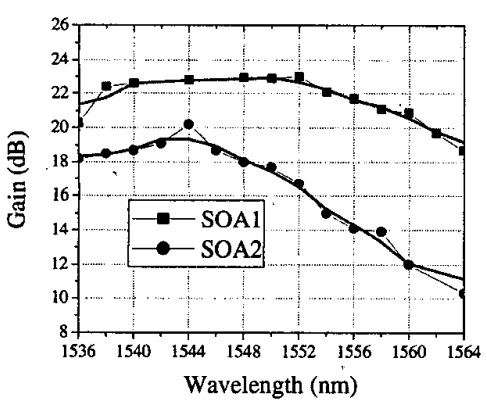

Fig. 1. Small signal gain of two SOAs with -15 $\mathrm{dBm}$ input power.

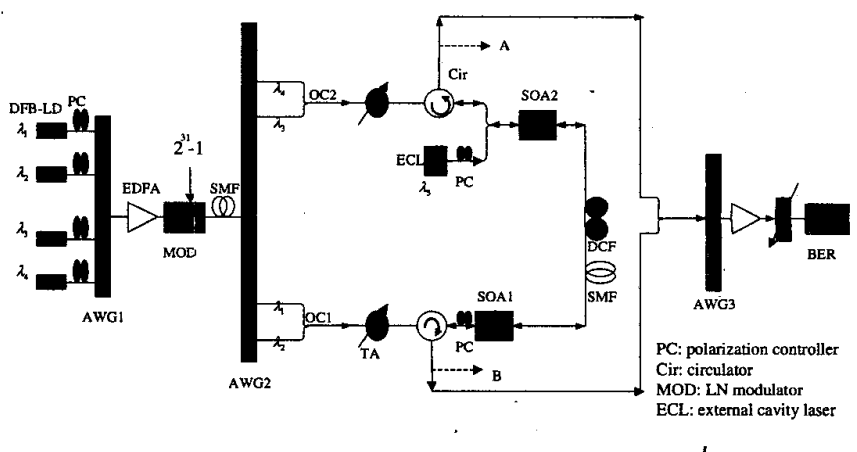

Fig.2 Experimental setup for $4 \times 10 \mathrm{~Gb} / \mathrm{s}$ bi-directional fiber transmission with two in-line SOAs.

The experimental setup is shown in Fig. 2. The outputs of four DFB lasers are multiplexed in an AWG. The operating wavelengths are $\lambda_{1}=1555.72 \mathrm{~nm}$ (channel 1), $\lambda_{2}=1557.33 \mathrm{~nm}$ (channel 2), $\lambda_{3}=1558.96 \mathrm{~nm}$ (channel 3) and $\lambda_{4}=1560.6 \mathrm{~nm}$ (channel 4). The amplified signals are modulated at $10 \mathrm{~Gb} / \mathrm{s}$ with a PRBS of sequence length $2^{31}-1$. The four-channel WDM signals are de-correlated by $25 \mathrm{~km}$ standard SMF. The SOAs used in the experiment are both $1200 \mu \mathrm{m}$ long and have less than $1 \mathrm{~dB}$ of polarization dependence and confinement factors 


\section{WDD58-3}

of 0.6. The lengths of the SMF and DCF are $44 \mathrm{~km}$ and $6 \mathrm{~km}$, respectively. The total loss of the fiber span is $12.3 \mathrm{~dB}$ and it is fully compensated at $1550 \mathrm{~nm}$. The holding light is generated by an ECL at $\lambda_{5}=1539 \mathrm{~nm}$. The power of the holding light into SOA 2 is $6 \mathrm{dBm}$, and the powers of the signals at $\lambda_{1}$ and $\lambda_{2}$ into SOA1 are both $13.8 \mathrm{dBm}$. The powers of the signals at $\lambda_{3}$ and $\lambda_{4}$ into SOA2 are $-16.7 \mathrm{dBm}$ and $-13.8 \mathrm{dBm}$, respectively. Because the gain of channel 3 is larger than that of channel 4 , the input power of channel 3 is smaller than that of channel 4. One polarization controller near SOA1 is used to keep the interference between signal and reflected signal to a minimum.

First we use a tunable attenuator to replace the SMF and DCF between the SOAs. The loss of the attenuator is adjusted to $2.3 \mathrm{~dB}$, which is the same as the loss of the fibers. The BER is measured and shown in Fig.3. The penalties with respect to the back-to-back BER are 1-2 $\mathrm{dB}$ at a BER of $10^{-9}$. The different input powers of signals and gain of SOAs lead to different penalties.

With fiber transmission, we can see that the penalties with respect to the back-to-back BER are 3-4 dB. It means that another $2 \mathrm{~dB}$ penalty are added when the signals are transmitted through the fibers. The added penalty is caused by the following two reasons: First, a negative chirp will be generated when the signals are transmitted through the SOAs and the negative chirp will cause some penalty in a fully dispersion compensated system. Second, Rayleigh Scattering and SBS caused by the strong CW holding light propagating in the fibers will degrade the system performance. In order to reduce the SBS effect, Ref. 2 has shown that we can modulate the holding light using a strong low frequency signal. It is important to emphasize that the BER measurements for both signals in the bi-directional transmission are performed simultaneously and very stable operation with no signs of error floor has been observed. Some eye diagrams are shown in Fig. 4. After the de-correlating SMF (back-to-back), a peaking eye diagram is obtained as shown in Fig. 4 (a). Because of the noise accumulation and inter-channel cross-talk fluctuation at "1"s can be seen in Fig. 4 (b) and (c).

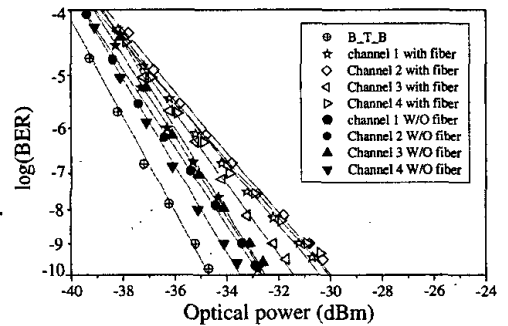

Fig. 3. BER for $4 \times 10 \mathrm{~Gb} / \mathrm{s}$ bi-directional fiber transmission with two in-line SOAs. W/O fiber: without fiber, B-T-B: back-to-back.

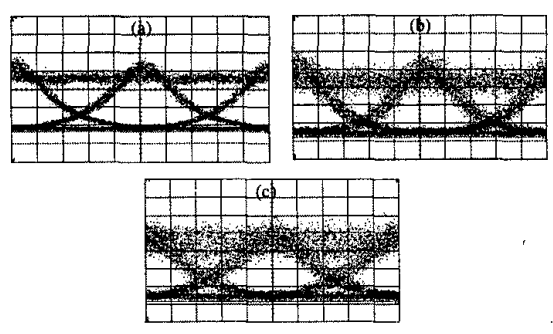

Fig. 4 Eye diagrams. (a) back-to-back, (b) channel 1 without fiber transmission, (c) channel 1 with fiber transmission.

For example, Fig. 5 shows the spectra at port A indicated in Fig. 2. Without SOAs means that the SOAs are taken away. It is clearly seen that the optical components of four-wave mixing in the SOA are very small, hence, their effect can be ignored. The total gain of the SOAs is approximately $21 \mathrm{~dB}$. Because the isolation of the circulators is not perfect, considerable power at channel 3 and 4 can be seen in Port A, but these unwanted signals will not affect our measurements at channel 1 and 2 because they can be effectively filtered away by AWG3. We can see that the SNR of all channels in a $0.1 \mathrm{~nm}$ optical bandwidth is larger than $30 \mathrm{~dB}$ after the signals have been transmitted through the fibers and SOAs.

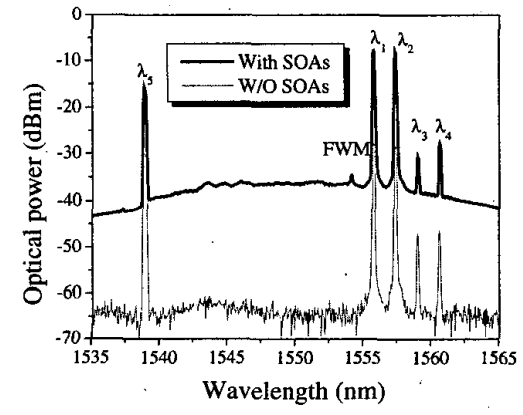

Fig. 5. Optical spectra measured at Port A.

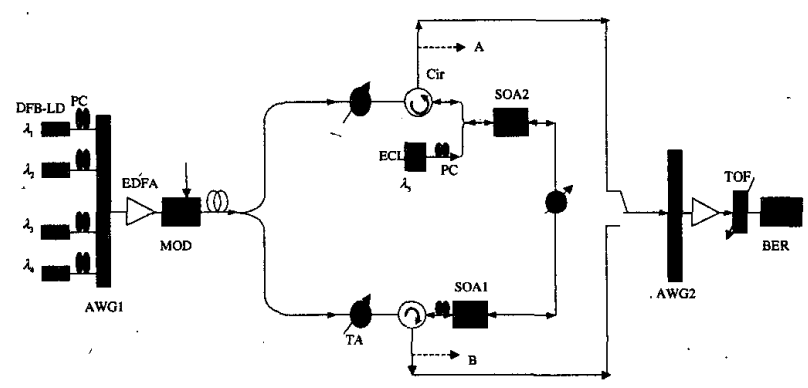

Fig.6. Experimental setup for $8 \times 10 \mathrm{~Gb} / \mathrm{s}$ bi-directional transmission with two in-line SOAs. 


\section{WDD58-4}

\section{B. $8 \times 10 \mathrm{~Gb} / \mathrm{s}$ bi-directional transmission with two in-line SOAs}

This experimental setup is almost the same as the one above. It is shown in Fig.6. Two sets of 4 WDM channels are obtained after the $3 \mathrm{~dB}$ optical coupler with the same set of wavelengths. One set is transmitted through the SOAs from SOA1 to SOA2, the other set from SOA2 to SOA1. The loss of the tunable attenuator between the SOAs is adjusted to be $6.3 \mathrm{~dB}$. In order to further suppress the cross talk in the SOAs, we let the holding light wavelength move to the gain peak of the SOAs. The holding light wavelength is near $1546 \mathrm{~nm}$.

First we investigate four channel uni-directional transmission. We measure the BER performance for signal transmission over only SOA1, the input power of the four channels is $-12 \mathrm{dBm}$ and the holding power is 4.4 $\mathrm{dBm}$. The measured BER result is shown in Fig. 7. Almost the same BER performance is obtained for the four channels and the penalty is smaller than $1 \mathrm{~dB}$. Then we measure the uni-directional transmission over two inline SOAs. It means that the signals are transmitted from SOA2 to SOA1 and no signals are transmitted in the opposite direction. The input powers into SOA2 are $-11.7,-11.7,-9.7$ and $-9.7 \mathrm{dBm}$ for channel 1, 2, 3 and 4, respectively. The holding light power is $4.4 \mathrm{dBm}$. The BER performance is measured and also shown in Fig. 8 . It is clearly seen that channel 3 has the smallest penalty. It should be pointed out that we did not optimise the input powers of the signals, and we only let channel 3 and 4 have a relatively large input power because channel 3 and 4 have small gain. After uni-directional transmission through two SOAs, the smallest penalty for channel 4 is $2 \mathrm{~dB}$ and the largest penalty for channel 1 or 2 is $5 \mathrm{~dB}$. The different penalties are caused by the facts that SOA2 has an un-flattened gain and the input signal powers are unequal.

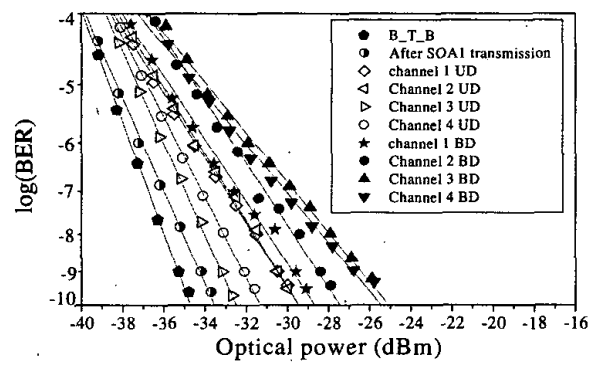

(a)

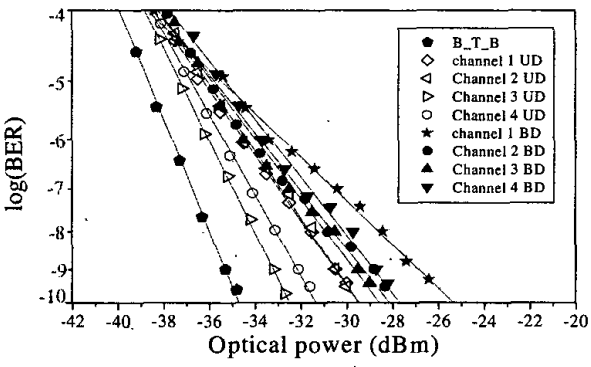

(b)

Fig.7. BER performance. UD: uni-directional transmission, BD: bi-directional transmission. (a) From SOA2 to SOA1, (b) from SOA1 to SOA2.

Then we investigate bi-directional transmission. The BER performance for signal transmission from SOA2 to SOA1 is shown in Fig. 7 (a). The input powers of channel 1, 2, 3 and 4 into SOA2 are -12, -12, -9.9 and -9.7 $\mathrm{dBm}$, respectively. The input powers of channel $1,2,3$ and 4 into SOA1 are $-19,-19,-16.9$ and $-16.2 \mathrm{dBm}$, respectively. The holding light is $8.4 \mathrm{dBm}$. The BER performance for signal transmission from SOA1 to SOA2 is shown in Fig. 7(b). The different penalties for all channels are caused by different gain of SOAs and unequal input powers. Error-free transmission is realized for all channels. The total gain of the SOAs is almost $8.2 \mathrm{~dB}$. Because the total gain of the SOAs is not large, we have not measured BER performance with fiber transmission. If the saturation output power of the SOAs can be further increased it would be possible for us to demonstrate $8 \times 10 \mathrm{~Gb} / \mathrm{s}$ bi-directional transmission over some fiber length. Ref. 4 has shown that a small confinement factor and short length are suitable for signal amplification, so, if such SOAs were available, better performance could be obtained. However, because of the large confinement factor and long cavity used here, we had to use a very large holding light power to suppress inter-channel cross-talk and that led to gain reduction of the SOAs.

\section{Conclusion}

We have demonstrated error-free $4 \times 10 \mathrm{~Gb} / \mathrm{s}$ WDM bi-directional transmission over $44 \mathrm{~km}$ SMF fiber combined with a suitable DCF and two in-line SOAs. We have also demonstrated error-free $8 \times 10 \mathrm{~Gb} / \mathrm{s} \mathrm{WDM}$ bi-directional transmission with two inline SOAs. The results suggest very promising applications of bidirectional transmission in short or medium scale optical networks with simple configuration because no isolators are necessary.

\section{Reference}

1: L. H. Spiekman, et al., IEEE Photon. Techon. Lett., Vol.12, No. 6, 2000: 717-719.

2. S. Banerjee, et al., 2000 Conference on Optical Fiber Communications, Baltimore, MD, paper: WM32.

3. G. Onishchukov, et al., Electron. Lett., Vol. 34, No. 16, 1998: 1597-1598.

4. D. Wolfson, IEEE Photon. Technol. Lett., Vol. 11, No. 11, 1999: 1494-1496.

5. S. Banerjee, et al., Proc. ECOC 99 , post-deadline paper, PD3-9, pp: 62-63, Nice, France.

6. M. Yoshino and K. Inoue, IEEE Photon. Technol. Lett., Vol. 8, No. 1, pp: 58-59, 1996.

7. J. Yu, et al., IEEE Photon. Technol. Lett., Vol. 12, No.6, 2000: 702-704. 